\title{
Changes in Calcaneal Bone Status Before and After Competitive Season in Female Collegiate Athletes
}

\author{
Shuichi Mizuno ${ }^{1}$ Kentaro TaI $^{2}$ Keiko MiYaHARA ${ }^{3}$ Kazuto OdA $^{3}$ Namie KoJIMA ${ }^{3}$ \\ Kayoko MATSUO ${ }^{3}$ Yoshitaka YoshIMURA $^{4}$ Kazuhide IIDE $^{5}$ Hiroyuki IMAMURA $^{3}$ \\ ${ }^{I}$ Mizuno Corporation \\ ${ }^{2}$ Department of International Tourism, Faculty of Human Sociology, Nagasaki International University \\ ${ }^{3}$ Department of Health and Nutrition, Faculty of Health Management, Nagasaki International University \\ ${ }^{4}$ Department of Food and Nutrition, Beppu University \\ ${ }^{5}$ Department of Physical Education, International Pacific University
}

\begin{abstract}
Background The purpose of this study was to examine changes in the speed of sound (SOS) in the calcaneus using a quantitative ultrasound device before and after the competitive season in female collegiate athletes.

Methods Thirty female collegiate athletes participated in the present study: 13 volleyball players, 9 tennis players and 8 karate practitioners. The SOS in the right calcaneus was measured using a quantitative ultrasound device and compared with the percentage of young adult mean. Dietary information was obtained with a food frequency questionnaire.

Resufts After the training period, lean body mass significantly increased in volleyball players, whereas there were no significant changes in tennis players and karate practitioners. Calcium intake significantly decreased in the volleyball and tennis players, whereas it did not change significantly in the karate practitioners. Energy, and vitamin $D$ and $K$ intakes did not change significantly in all 3 groups. After the training period, the SOS and the percentage of young adult mean significantly increased in the volleyball and tennis players, whereas they did not change significantly in the karate practitioners.
\end{abstract}

Conclusion These results suggest that volleyball and tennis practice could increase the SOS in the calcaneus after the competitive season.

(HEP. 2015; 42: 435-438.)

Key words quantitative ultrasound, speed of sound, calcaneal bone status, volleyball, tennis, karate

\section{Introduction}

When examining the effects of physical exercise on bone mineral density (BMD), the bones in the foot are important for evaluation as they bear the greatest forces due to gravity during exercise $^{1)}$. In a previous study ${ }^{2)}$, we evaluated the speed of sound (SOS) in the calcaneus using a quantitative ultrasound (QUS) device, compared SOS among female collegiate athletes (16 tennis and 30 volleyball players) and 45 controls, and examined the relationship between SOS and physical characteristics, namely, maximal oxygen uptake, and nutrient and dietary intake. The results showed that the tennis and volleyball players had significantly higher mean SOS than the controls. The results did not change even after the mean values had been adjusted for body height and weight.

A QUS device involves no X-ray exposure ${ }^{1)}$, so it is easier to examine longitudinal changes of bone status. The purpose of this study was to examine changes in calcaneal bone status by means

Received: March 30, 2015, Accepted: May 8, 2015

Corresponding author: Hiroyuki Imamura

${ }^{3}$ Address; Department of Health and Nutrition, Faculty of Health

Management, Nagasaki International University

2825-7 Huis Ten Bosch, Sasebo-shi, Nagasaki 859-3298, Japan

TEL: +0956-39-2020, FAX: +0956-39-3111

E-mail: himamura@niu.ac.jp of SOS before and after the competitive season in female collegiate athletes.

\section{Materials and methods}

\section{Subjects}

The subjects were 30 female collegiate athletes: 13 volleyball players, 9 tennis players and 8 karate practitioners. The mean $( \pm$ SD) duration of experience of playing volleyball, tennis, and karate among the players were $9.9 \pm 1.5,9.3 \pm 2.7$, and $12.8 \pm 2.2$ y, respectively. The study protocol was approved by the University Ethics Committee. Informed consent was obtained from each subject.

Measurements and dietary information

All measurements and dietary information were obtained before (April) and after (December) the competitive season. Weight and height were measured to the nearest $0.1 \mathrm{~kg}$ and 0.1 $\mathrm{cm}$, respectively. The body mass index (BMI) was calculated as weight $/$ height ${ }^{2}\left(\mathrm{~kg} / \mathrm{m}^{2}\right)$. The biceps brachii, triceps brachii, subscapular and suprailiac skinfold thicknesses were measured with a Harpenden caliper on the right side of the body with the subject in a standing position and are expressed as the mean of 3 consecutive measurements. The average of 3 measurements at each site was used to calculate the body density ${ }^{3)}$, percent body fat (\%Fat) and lean body mass (LBM) $)^{4}$.

QUS measurements of the right calcaneus were performed 
(CM-100, Canon Lifecare Solutions Inc., Osaka, Japan). After cleaning the skin with ultrasound gel (Parker Laboratories, Inc., New Jersey, USA), the subject's heel was positioned in a small measurement device. An ultrasonic wave was transmitted through the heel and detected by a receiving transducer. SOS was measured using this device, which refers to the velocity of the ultrasonic wave as it passes through the heel, and was compared with the percentage of young adult mean (\%YAM).

All subjects were interviewed by experienced dietitians using a food frequency questionnaire (FFQ), which is based on 29 food groups and 10 types of cooking, for estimating the energy and nutrient intakes of each subject during the past 1 to 2 months ${ }^{5}$. The FFQ was validated by a comparison with weighed dietary records for 7 continuous days ${ }^{6}$. From the FFQ, the mean daily intake of total energy and nutrients was calculated according to the Tables of the Japanese Foodstuff Composition ${ }^{7}$. Information on nutrient supplements and/or on the diet was obtained via a self-administered questionnaire. The accuracy of the questionnaire was checked by individual interviews.

\section{Statistical analysis}

SPSS statistical software 22.0J (Chicago, IL) was used to analyze the data. Descriptive statistics are presented as means and SD. Because sample size in each group was small, non-parametric statistics were used. Data before and after the experimental period were analyzed by paired Mann-Whitney U test. Two-sided $\mathrm{p}<0.05$ was considered to be statistically significant.

\section{Results}

The characteristics of the subjects are shown in Table 1. After the training period, LBM significantly increased in volleyball players, whereas there were no significant changes in tennis players and karate practitioners.

The selected energy and nutrient intakes of the subjects are shown in Table 2. Calcium intake significantly decreased in the volleyball and tennis players, whereas it did not change significantly in the karate practitioners. Energy, vitamin D and K intakes did not change significantly among the 3 groups.

The SOS and \%YAM of the subjects are shown in Fig. 1 and 2, respectively. After the training period, the volleyball and tennis

Table 1 Characteristics of the subjects

\begin{tabular}{|c|c|c|c|c|c|c|c|c|c|}
\hline & \multicolumn{2}{|c|}{ volleyball $(n=13)$} & & \multicolumn{2}{|c|}{ tennis $(n=9)$} & \multicolumn{4}{|c|}{ karate $(n=8)$} \\
\hline & Before & After & & Before & After & & Before & After & \\
\hline Height (cm) & $164.0 \pm 5.2$ & $164.3 \pm 5.2$ & * & $156.5 \pm 6.3$ & $156.7 \pm 6.3$ & * & $160.6 \pm 5.9$ & $161.2 \pm 5.8$ & * \\
\hline BMI $\left(\mathrm{kg} / \mathrm{m}^{2}\right)$ & $22.6 \pm 1.4$ & $22.5 \pm 1.4$ & & $21.8 \pm 1.9$ & $21.7 \pm 2.2$ & & $23.2 \pm 4.0$ & $23.3 \pm 4.1$ & \\
\hline$\%$ Fat & $26.9 \pm 1.6$ & $25.3 \pm 2.5$ & & $27.0 \pm 2.9$ & $26.8 \pm 4.4$ & & $29.9 \pm 4.1$ & $29.3 \pm 6.1$ & \\
\hline Fat mass (kg) & $16.4 \pm 2.0$ & $15.4 \pm 2.4$ & & $14.6 \pm 2.9$ & $14.5 \pm 3.8$ & & $18.3 \pm 6.2$ & $18.3 \pm 7.2$ & \\
\hline
\end{tabular}

mean \pm SD. BMI: Body mass Index. * $p<0.05$ Before vs After.

Table 2 Energy and selected micronutrient intakes of the subjects

\begin{tabular}{|c|c|c|c|c|c|c|c|c|}
\hline & \multicolumn{2}{|c|}{ volleyball $(n=13)$} & & \multicolumn{2}{|c|}{ tennis $(n=9)$} & & \multicolumn{2}{|c|}{ karate $(n=8)$} \\
\hline & Before & After & & Before & After & & Before & After \\
\hline energy (kcal) & $1,713 \pm 445$ & $1,683 \pm 449$ & & $1,817 \pm 154$ & $1,515 \pm 365$ & & $1,612 \pm 224$ & $1,662 \pm 284$ \\
\hline calcium (mg) & $461 \pm 147$ & $370 \pm 149$ & * & $487 \pm 117$ & $341 \pm 101$ & * & $509 \pm 171$ & $424 \pm 161$ \\
\hline vitamin $\mathrm{D}(\mu \mathrm{g})$ & $2.4 \pm 1.2$ & $2.0 \pm 1.4$ & & $2.3 \pm 1.1$ & $1.3 \pm 0.6$ & & $2.6 \pm 1.0$ & $2.3 \pm 1.0$ \\
\hline vitamin $\mathrm{K}(\mu \mathrm{g})$ & $160.3 \pm 73.7$ & $144.4 \pm 48.0$ & & $187.9 \pm 69.7$ & $133.6 \pm 60.2$ & & $175.8 \pm 36.8$ & $129.8 \pm 48.0$ \\
\hline
\end{tabular}

mean \pm SD. ${ }^{*} p<0.05$ Before vs After.

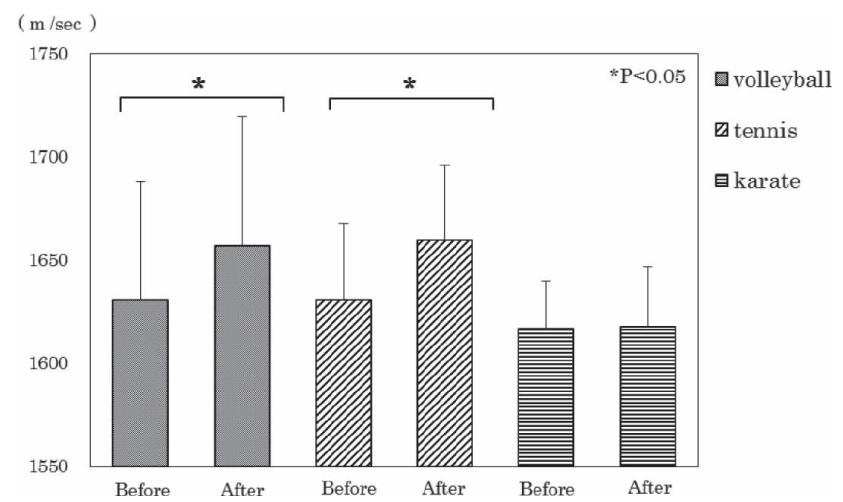

Fig. 1 Changes in the speed of sound in the right calcaneus after the competitive season.

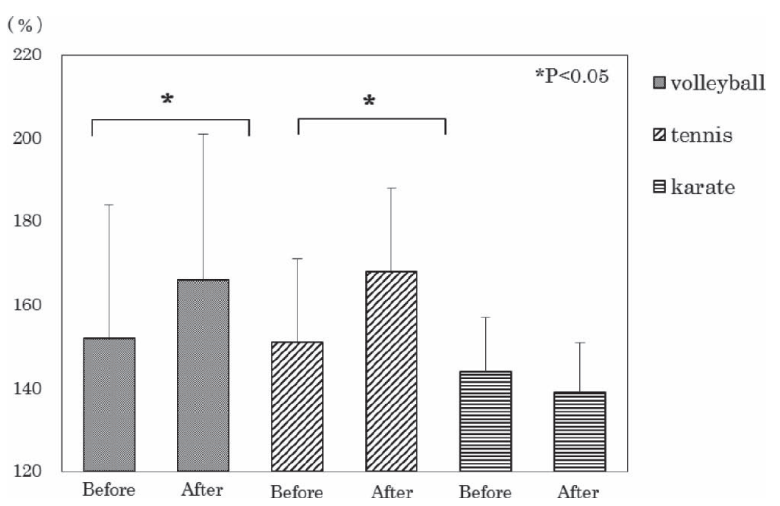

Fig. 2 Changes in percentage of young adult mean after the competitive season. 
players exhibited significant increases of SOS and \%YAM, whereas the karate practitioners did not show significant changes.

\section{Discussion}

It has been shown that athletes involved in weight-bearing activities $^{8-10)}$ have greater BMD than non-active controls. In these studies, BMD was measured at various sites with dual-energy X-ray absorptiometry (DXA), which remains the optimal method for evaluating BMD. In the present study, as well as our previous study $^{2)}$, we evaluated the SOS of the calcaneus using a QUS device, as opposed to the conventional measurement by DXA, for the following reasons. First, QUS is a portable and practical machine that measures bone status and involves no X-ray exposure $^{1)}$, so it is easier to examine longitudinal changes of bone status. Second, a relatively high correlation coefficient $(r=0.76)$ between SOS and BMD measured by DXA at the heel assessed at the location corresponding to that of the QUS measurement has been reported, and SOS value was significantly lower in fracture patients than in participants without fractures ${ }^{11)}$. Third, it has further been shown that SOS of the calcaneus is comparable to DXA in identifying subjects with vertebral fractures ${ }^{12,13}$.

Regarding the BMD of volleyball players, Creighton et al. ${ }^{14)}$ evaluated BMD and compared 3 impact groups of female athletes: high impact (High, basketball and volleyball, $n=14$ ), medium impact (Med, soccer and track, $n=13$ ) and nonimpact (Non, swimming, $\mathrm{n}=7$ ), with sedentary age-matched controls (Con, $\mathrm{n}=7$ ). The results showed that adjusted BMD (covariates: BMI, weight, and calcium and calorie intakes) was significantly higher at the femoral neck and trochanter in the High group than in the Non and Con groups. Total body BMD was significantly higher in the High group than in the Med, Non and Con groups and was higher in the Med group than in the Non and Con groups. Alfredson et al. ${ }^{15)}$ compared the BMD of female volleyball players with age- and height-matched controls. The results showed that the volleyball players had a significantly higher BMD of the total body, lumbar spine, femoral neck, Ward's triangle, trochanter, nondominant femur and humerus.

Regarding the BMD of tennis players, Calbet et al. ${ }^{16)}$ compared 9 male tennis players with 17 inactive subjects. The results showed that adjusted BMD (covariates: body weight and height) was significantly higher at the lumbar spine and femoral neck than in the inactive subjects. Other studies compared the BMD of dominant and nondominant forearms in tennis players and found a marked side-to-side difference in favor of the dominant fore$\operatorname{arm}^{17,18)}$

Regarding the BMD of karate practitioners, Andreoli et al. ${ }^{19)}$ compared the BMD of 21 judo, 14 karate and 24 water polo male practitioners. The results showed that the judo and karate practitioners had significantly higher total BMD than the water polo group and 12 age-matched controls. In addition, Drozdzowska et $a l{ }^{20)}$ examined skeletal status assessed by QUS at the hand phalanges in 226 karate practitioners (7 to 61 years of age). The results showed that, up to age 18 , there were no significant differences between the karate practitioners and controls, while afterwards, up to age 35 , the difference increased and then stabi- lized after age 35. These authors stated that longer duration, higher frequency and earlier start of physical training positively influenced the skeletal status and concluded that karate is a sport with a positive influence on the skeletal status, with the most significant benefits occurring in adults.

The results of the above-mentioned studies on volleyball ${ }^{14,15)}$, tennis ${ }^{16-18)}$ and karate ${ }^{19,20)}$ indicate that athletes who participate in high-impact sports have higher BMD than controls. However, these are cross-sectional studies, which do not permit the assessment of causality owing to the uncertain temporality of the association.

In a previous cross-sectional study ${ }^{2}$, we evaluated the SOS of the calcaneus using a QUS device, compared the SOS among female collegiate tennis players, volleyball players and 45 controls, and examined the relationship between SOS and physical characteristics, maximal oxygen uptake, and nutrient and dietary intakes. The results showed that the tennis and volleyball players had significantly higher mean SOS than the controls. The results did not change even after the mean values had been adjusted for body height and weight. In the present study, we further evaluated the SOS of the calcaneus and compared it before and after the competitive season. The results showed that the volleyball and tennis players exhibited significant increases in SOS after the competitive season, whereas the karate practitioners showed no change. As far as we are aware, this is the first study to show increased SOS of the calcaneus after the competitive season in female volleyball and tennis players.

The limitations of the present study need to be mentioned. The sample size was small and a control group was not included. However, in the present study, the SOS of the karate practitioners did not change. A few years before the present study, we obtained similar results in karate practitioners. The mean values of SOS measured in April and July were 1,632 \pm 40 and 1,624 $\pm 31 \mathrm{~m} / \mathrm{sec}$, respectively. We have also obtained similar results during the last 3 years, so we consider that karate practitioners can be used as a control group. Thus, further studies with a large number of subjects including a control group are needed to confirm our results.

In conclusion, these results suggest that volleyball and tennis practice could increase the SOS of the calcaneus after the competitive season.

\section{Acknowledgements}

The authors declare that they have no competing interests.

This study was supported by grants from Nagasaki International University.

The authors state that they have no Conflict of Interest (COI).

\section{REFERENCES}

1) Nohara $T$, Ueda M, Ohta A, Sugimoto T. Correlation of body growth and bone mineral density measured by ultrasound densitometry of the calcaneus in children and adolescents. Tohoku J Exp Med 2009; 219: 63-9.

2) Mizuno S, Miyahara K, Kojima N, Oda K, Matsuo K, Iide K, et al. Factors related with calcaneal bone mineral density in 
female collegiate athletes. HEP 2014; 41(3): 411-7 (in Japanese).

3) Durnin JVGA, Womersley J. Body fat assessed from total body density and its estimation from skinfold thickness: measurements on 481 men and women aged 16 to 72 years. Br J Nutr 1974; 32: 77-97.

4) Brozek J, Grande F, Anderson JT, Keys A. Densitometric analysis of body composition: Revision of some quantitative assumptions. Ann NY Acad Sci 1963; 110: 113-40.

5) Yoshimura Y, Takahashi K. Excel Eiyo-kun (nutrition) Food Frequency Questionnaire Based on Food Groups FFQg (computer manual and software). Tokyo: Kenpakusya, 2001 (in Japanese).

6) Takahashi K, Yoshimura Y, Kaimoto T, Kunii D, Komatsu T, Yamamoto S. Validation of a food frequency questionnaire based on food groups for estimating individual nutrient intake. Jpn J Nutr 2001; 59: 221-32 (in Japanese with English abstract).

7) The Resources Council of the Science and Technology Agency (ed). The $5^{\text {th }}$ Revised Edition of Tables of Japanese Foodstuff Composition. Tokyo: Ishiyaku Press, 2001 (in Japanese).

8) Morgan AL, Jarrett JW. Markers of bone turnover across a competitive season in female athletes: a preliminary investigation. J Sports Med Phys Fitness 2011; 51: 515-24.

9) Prouteau S, Pelle A, Collomp K, Benhamou L, Corteix D. Bone density in elite judoists and effects of weight cycling on bone metabolic balance. Med Sci Sports Exerc 2006; 38: 694-700.

10) Andreoli A, Monteleone M, Van Loan M, Promenzio L, Tarantino U, De Lorenzo A. Effects of different sports on bone density and muscle mass in highly trained athletes. Med Sci Sports Exerc 2001; 33: 507-11.

11) Graafmans WC, Van Lingen $A$, Ooms ME, Bezemer PD, Lips $P$. Ultrasound measurements in the calcaneus: precision and its relation with bone mineral density of the heel, hip, and lumbar spine. Bone 1996; 19: 97-100.

12) Glüer CC, Eastell R, Reid DM, Felsenberg D, Roux C, Barkmann $\mathrm{R}$, et al. Association of five quantitative ultrasound devices and bone densitometry with osteoporotic vertebral fractures in a population-based sample: the OPUS Study. J Bone Miner Res 2004; 19: 782-93.

13) Hartl F, Tyndall A, Kraenzlin M, Bachmeier C, Gückel C, Senn $\mathrm{U}$, et al. Discriminatory ability of quantitative ultrasound parameters and bone mineral density in a population-based sample of postmenopausal women with vertebral fractures: results of the Basel Osteoporosis Study. J Bone Miner Res 2002; 17: 321-30.

14) Creighton DL, Morgan AL, Boardley D, Brolinson G. Weightbearing exercise and markers of bone turnover in female athletes. J Appl Physiol 2001; 90: 565-70.

15) Alfredson P, Nordström P, Lorentzon R. Bone mass in female volleyball players: A comparison of total and regional bone mass in female volleyball players and nonactive females. Calcif Tissue Int 1997; 60: 338-42.

16) Calbet JA, Moysi JS, Dorado C, Rodríguez LP. Bone mineral content and density in professional tennis players. Calcif Tissue Int 1998; 62: 491-6.

17) Haapasalo H, Kannus P, Sievanen H, et al. Effect of long-term unilateral activity on bone mineral density of female junior tennis players. J Bone Miner Res 1998; 13: 310-9.

18) Ducher G, Jaffré C, Arlettaz A, Benhamou C-L, Courteix D. Effects of long-term tennis playing on the muscle-bone relationship in the dominant and nondominant forearms. Can J Appl Physiol 2005; 303-17.

19) Andreoli A, Monteleone M, Van Loan M, Promenzio L, Tarantino U, De Lorenzo A. Effects of different sports on bone density and muscle mass in highly trained athletes. Med Sci Sports Exerc 2001; 33: 507-11.

20) Drozdzowska B, Münzer U, Adamczyk P, Pluskiewicz W. Skeletal status assessed by quantitative ultrasound at the hand phalanges in karate training males. Ultrasound in Med \& Biol 2011; 37: 214-19. 